International Journal of Pure and Applied Mathematics

Volume 86 No. 3 2013, 527-535

ISSN: 1311-8080 (printed version); ISSN: 1314-3395 (on-line version)

url: http://www.ijpam.eu

doi: http://dx.doi.org/10.12732/ijpam.v86i3.7

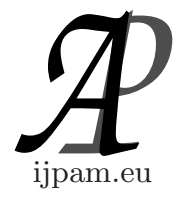

\title{
EXISTENCE AND CHARACTERIZATION OF FARTHEST POINTS IN NORMED LINEAR SPACES
}

\author{
R. Saravanan ${ }^{1}$, R. Vijayaragavan ${ }^{2} \S$ \\ ${ }^{1}$ School of Information Technology and Engineering \\ VIT University \\ Vellore, 632 014, Tamilnadu, INDIA \\ ${ }^{2}$ Applied Analysis Division \\ School of Advanced Sciences \\ VIT University, Vellore, 632 014, Tamilnadu, INDIA
}

\begin{abstract}
This paper delineates existence and characterization of a set of farthest points in normed linear spaces.
\end{abstract}

AMS Subject Classification: 41A50, 41A52, 41A65

Key Words: best approximation, farthest points, existence, characterization, normed linear spaces

\section{Introduction}

The problem of farthest points in normed linear spaces has been studied by Franchetti and Singer [4]. They obtained some results on characterization and existence of farthest points in normed linear spaces in terms of bounded linear functionals. Asplund [2] and Edelstein [3] have considered the farthest points of sets in uniformly convex Banach spaces. Several results related with farthest

Received: March 27, 2013

(C) 2013 Academic Publications, Ltd. url: www.acadpubl.eu

${ }^{\S}$ Correspondence author 
points in the context of normed linear space and metric space were obtained by Panda, et.al. [7] and Ahuja, et.al. [1] respectively. Section 2 provides the existence of farthest points in normed linear spaces. Some characterizations of farthest points with respect to uniform norm are established in Section 3.

\section{Existence of Farthest Points}

Definition 2.1. Let $G$ be a bounded subset of a real normed linear space $X$ and $x \in X$. An element $g_{0} \in G$ is called a farthest point to $x \in X$ from $G$ if for every $g \in G,\left\|x-g_{0}\right\| \geq\|x-g\|$, i.e.

$$
\left\|x-g_{0}\right\|=\sup _{g \in G}\|x-g\| .
$$

The set of all farthest points to $x$ from $G$ is denoted by $F_{G}(x)$.

Example 2.2. Let $X=\mathbb{R} \times \mathbb{R}, x=(a, b)$ in $X$ we define a $l_{\infty}$ - norm on $X$ as follows:

$$
\|(a, b)\|_{\infty}=\max \{|a|,|b|\}, a, b \in \mathbb{R} .
$$

Let $G=\left\{\left(g_{1}, g_{2}\right):-3 \leq g_{1} \leq 3\right.$ and $\left.g_{2}=0\right\}$ and $x=(5,0)$. Then we can see that $g_{0}=(-3,0) \in F_{G}(x)$.

Let $G=\left\{\left(g_{1}, g_{2}\right): g_{1}=0\right.$ and $\left.-5 \leq g_{2} \leq 5\right\}$ and $x=(0,1)$.

Then clearly $g_{0}=(0,-5) \in F_{G}(x)$.

Definition 2.3. Let $G$ be a bounded subset of a real normed linear space $X$ and $x \in X$. An element $g_{0} \in G$ is called a strongly unique farthest point to $X$ from $G$, if there exists a constant $K_{x}>0$ such that for every $g \in G$,

$$
\left\|x-g_{0}\right\| \geq\|x-g\|+K_{x}\left\|g-g_{0}\right\| .
$$

Theorem 2.4. Let $G$ be a compact subset in a normed linear space $X$. Then for every $x \in X$, there exists a farthest point from $G$.

Proof. Let $d=\sup _{g \in G}\|x-g\|$. Thus by definition of supremum there exists a sequence $\left\{g_{n}\right\}$ in $G$ such that

$$
\left\|x-g_{n}\right\| \rightarrow d \text { as } n \rightarrow \infty
$$

Since $G$ is compact, there exists a sub sequence $\left\{g_{n_{k}}\right\}$ such that $g_{n_{k}} \rightarrow g_{0}$, for some $g_{0} \in G$. 
Now we will show that $g_{0}$ is a farthest point to $x$ from $G$.

Now

$$
\begin{gathered}
\left\|x-g_{n_{k}}\right\| \leq\left\|x-g_{0}\right\|+\left\|g_{0}-g_{n_{k}}\right\| \\
\Rightarrow\left\|x-g_{0}\right\| \geq\left\|x-g_{n_{k}}\right\|-\left\|g_{0}-g_{n_{k}}\right\| \\
\rightarrow d \text { as } k \rightarrow \infty .
\end{gathered}
$$

Hence

$$
\left\|x-g_{0}\right\| \geq d
$$

since $g_{0} \in G,\left\|x-g_{0}\right\| \leq d$.

Therefore $\left\|x-g_{0}\right\|=d$.

The proof of the following corollaries is obvious.

Corollary 2.5. Let $G$ be a closed and bounded finite dimensional set in a normed linear space $X$. Then for every $x \in X$, there exists a farthest point in $G$.

Corollary 2.6. Let $G$ be a complete and totally bounded subset of a normed linear space $X$. Then for every $x \in X$, there exists a farthest point in $G$.

Corollary 2.7. Let $G$ be a closed subset of a compact normed linear space $X$. Then for every $x \in X$, there exists a farthest point in $G$.

Corollary 2.8. Let $f$ be a continuous mapping from a normed linear space $X$ into another normed linear space $Y$. Let $G$ be a compact set in $X$. Then for every $y \in Y$, there exists a farthest point from $f(X)$.

Corollary 2.9. Let $f$ be a continuous mapping from a normed linear space $X$ into another finite dimensional normed linear space $Y$. Let $G$ be a compact set in $X$. Then for every $y \in Y$, there exists a farthest point from convex hull of $f(X)$.

Corollary 2.10. Let $G$ be a totally bounded subset of a Banach space $X$. Then for every $x \in X$, there exists a farthest point in $G$.

Theorem 2.11. Every strongly unique farthest point is a unique farthest point.

Proof. Let $X$ be a normed linear space, $G$ be a bounded subset of $X, x \in X$ and $g_{0} \in G$ be a strongly unique farthest point to $x$ from $G$.

Claim. $g_{0}$ is a unique farthest point. If possible assume that $g_{1} \in G$ is a farthest point to $x$ from $G$.

Then $\left\|x-g_{1}\right\| \geq\|x-g\|$ for every $g \in G$ 
$\Rightarrow\left\|x-g_{1}\right\| \geq\left\|x-g_{0}\right\|$.

Since $g_{0}$ is a strongly unique farthest point to $x$ from $G$, we have

$$
\begin{gathered}
\left\|x-g_{0}\right\| \geq\|x-g\|+t\left\|g-g_{0}\right\| \text { for every } g \in G \text { and for some } \mathrm{t}>0 \\
\Rightarrow\left\|x-g_{0}\right\| \geq\left\|x-g_{1}\right\|+t\left\|g_{1}-g_{0}\right\| .
\end{gathered}
$$

From (2.1) and (2.2) it follows that

$$
\begin{gathered}
\left\|x-g_{1}\right\| \geq\left\|x-g_{1}\right\|+t\left\|g_{1}-g_{0}\right\| . \\
\Rightarrow t\left\|g_{1}-g_{0}\right\|=0 \\
\Rightarrow g_{1}=g_{0},
\end{gathered}
$$

since $t>0$.

\section{Characterization of Farthest Points}

The characterization of farthest points are obtained in this section.

Definition 3.1. Let $f \in C[a, b]$. Then uniform norm or $L_{\infty}$-norm $f$ is defined by

$$
\|f\|_{\infty}=\sup _{x \in[a, b]}|f(x)| .
$$

The following notation is used in the subsequent result. The set of extreme points of a function $f \in C[a, b]$ is defined by

$$
E(f)=\left\{x \in[a, b]:|f(x)|=\|f\|_{\infty}\right\} .
$$

The following results provide a characterization of farthest points with respect to uniform norm corresponding to Kolmogorov [5].

Theorem 3.2. Let $G$ be a compact subset of $C[a, b]$, containing $0, f \in$ $C[a, b]$ and $g_{0} \in G$. The following statements are equivalent:

(i) the function $g_{0}$ is a farthest point to $f$ from $G$

(ii) for every function $g \in G$,

$$
\min _{t \in E(f-g)}(f(t)-g(t))\left(g_{0}(t)-g(t)\right) \leq 0 .
$$


Proof. (ii) $\Rightarrow$ (i). Suppose that (ii) holds and let $g \in G$ be given. By (ii) there exists a point $t \in E\left(f-g_{0}\right)$ such that

$$
(f(t)-g(t))\left(g_{0}(t)-g(t)\right) \leq 0 .
$$

Then we have

$$
\begin{aligned}
\left\|f-g_{0}\right\|_{\infty} & \geq\left|f(t)-g_{0}(t)\right| \\
& =\left|f(t)-g(t)-\left(g_{0}(t)-g(t)\right)\right| \\
& =|f(t)-g(t)|+\left|g_{0}(t)-g(t)\right| \\
& \geq|| f-g \|_{\infty} .
\end{aligned}
$$

Which shows that (i) holds.

(i) $\Rightarrow$ (ii). Suppose that (ii) fails. Then there exists a function $g_{1} \in G$ such that for all $t \in E\left(f-g_{1}\right)$,

$$
\left(f(t)-g_{1}(t)\right)\left(g_{0}(t)-g_{1}(t)\right)>0 .
$$

Claim. $E\left(f-g_{1}\right)$ is compact.

Since $E\left(f-g_{1}\right)$ is a bounded subset of $\mathbb{R}$, it is sufficient to show that $E\left(f-g_{1}\right)$ is closed.

Let $\left\{t_{n}\right\} \in E\left(f-g_{1}\right)$ such that $t_{n} \rightarrow t$. Then

$$
\begin{aligned}
\left|f(t)-g_{1}(t)\right| & =\left|f\left(\lim t_{n}\right)-g_{1}\left(\lim t_{n}\right)\right| \\
& =\lim \left|f\left(t_{n}\right)-g_{1}\left(t_{n}\right)\right| \\
& =\lim \left|f-g_{1}\right|_{\infty} \\
& =\left\|f-g_{1}\right\|_{\infty}
\end{aligned}
$$

Thus $t \in E\left(f-g_{1}\right)$. Hence the claim is true.

Since $E\left(f-g_{1}\right)$ is compact, there exists a real number $c>0$ such that for all $t \in E\left(f-g_{1}\right)$,

$$
\left(f(t)-g_{1}(t)\right)\left(g_{0}(t)-g_{1}(t)\right)>C .
$$

Moreover, there exists a neighborhood $U$ of $E\left(f-g_{1}\right)$ such that for all $t \in U$,

$$
\left(f(t)-g_{1}(t)\right)\left(g_{0}(t)-g_{1}(t)\right)>\frac{c}{2}
$$

and

$$
\left|f(t)-g_{1}(t)\right| \geq \frac{1}{2}|| f-g_{1} \|_{\infty} .
$$


Since $[a, b] \backslash U$ is compact, $\max _{t \in[a, b] \backslash U}\left|f(t)-g_{1}(t)\right|$ exists. It is clear that for all $t \in[a, b] \backslash U$,

$$
\begin{aligned}
\left|f(t)-g_{1}(t)\right| & \leq \max _{t \in[a, b] \backslash U}\left|f(t)-g_{1}(t)\right| \\
& =\left\|f-g_{1}\right\|_{\infty}-d,
\end{aligned}
$$

where $d=\left\|f-g_{1}\right\|_{\infty}-\max _{t \in[a, b] \backslash U}\left|f(t)-g_{1}(t)\right|$.

Thus for all $t \in[a, b] \backslash U$,

$$
\left|f(t)-g_{1}(t)\right| \leq\left\|f-g_{1}\right\|_{\infty}-d .
$$

By multiplying $\left(g_{0}-g_{1}\right)$ with an appropriate positive factor, we may assume without loss of generality that

$$
\left\|g_{0}-g_{1}\right\|_{\infty} \leq \min \left\{d, \frac{1}{2}\left\|f-g_{1}\right\|_{\infty}\right\}
$$

Case (i) Let $t \in[a, b] \backslash U$. Then

$$
\begin{aligned}
\left|f(t)-g_{0}(t)\right| & \leq\left|f(t)-g_{1}(t)\right|+\left|g_{0}(t)-g_{1}(t)\right| & \\
& \leq\left\|f-g_{1}\right\|_{\infty}-d+\left\|g_{0}-g_{1}\right\|_{\infty} & \text { by }(3.3) \\
& <\left\|f-g_{1}\right\|_{\infty} . & \text { by }(3 .
\end{aligned}
$$

Case (ii) Let $t \in U$. Then

$$
\begin{aligned}
\left|f(t)-g_{0}(t)\right| & =\left|\left(f(t)-g_{1}(t)\right)-\left(g_{0}(t)-g_{1}(t)\right)\right| \\
& =|| f(t)-g_{1}(t)|-| g_{0}(t)-g_{1}(t)|| \\
& =\left|f(t)-g_{1}(t)\right|-\left|g_{0}(t)-g_{1}(t)\right| \text { by }(3.2) \text { and }(3.4) \\
& <\left|f(t)-g_{1}(t)\right| \\
& =|| f-g_{1} \|_{\infty} .
\end{aligned}
$$

Thus for all $t \in[a, b]$,

$$
\left|f(t)-g_{0}(t)\right|<\left\|f-g_{1}\right\|_{\infty}
$$

This implies that

$$
\left\|f-g_{0}\right\|_{\infty}<\left\|f-g_{1}\right\|_{\infty}
$$

which shows that $g_{0}$ is not a farthest point to $f$ from $G$, a contradiction.

Theorem 3.3. Let $G$ be a compact subset of $C[a, b]$ containing $0, f \in$ $C[a, b] \backslash G$ and $g_{0} \in G$.

Consider the following statements. 
(i) the function $g_{0}$ is a strongly unique farthest point to $f$ from $G$.

(ii) for $g \in G$,

$$
\min _{t \in E(f-g)}(f(t)-g(t))\left(g_{0}(t)-g(t)\right)<0
$$

(iii) there exists a constant $K_{f}>0$ such that for every $g \in G$,

$$
\min _{t \in E(f-g)}(f(t)-g(t))\left(g_{0}(t)-g(t)\right) \leq-K_{f}\|f-g\|_{\infty}\left\|g_{0}-g\right\|_{\infty} .
$$

Then (i) $\Leftrightarrow$ (iii) and (iii) $\Rightarrow$ (ii). If $G \backslash\left\{g_{0}\right\}$ is compact, then (ii) $\Rightarrow$ (iii).

Proof. (iii) $\Rightarrow$ (i). Suppose that (iii) holds and let $g \in G$ be given.

By (iii) there exists a point $t \in E(f-g)$ such that

$$
(f(t)-g(t))\left(g_{0}(t)-g(t)\right) \leq-K_{f}\|f-g\|_{\infty}\left\|g-g_{0}\right\|_{\infty} .
$$

Then it follows that

$$
\begin{aligned}
\left\|f-g_{0}\right\|_{\infty} & \geq\left|f(t)-g_{0}(t)\right| \\
& =\left|(f(t)-g(t))-\left(g_{0}(t)-g(t)\right)\right| \\
& =|f(t)-g(t)|+\left|g_{0}(t)\right|-g(t) \mid \\
& \geq\|f-g\|_{\infty}+K_{f} \frac{|| f-g\left\|_{\infty}\right\| g_{0}-g \|_{\infty}}{|f(t)-g(t)|} \\
& =\|f-g\|_{\infty}+K_{f}\left\|g_{0}-g\right\|_{\infty} .
\end{aligned}
$$

(i) $\Rightarrow$ (iii). Suppose that (iii) fails, then there exists a function $g_{1} \in G$ such that for all $t \in E\left(f-g_{1}\right)$,

$$
\left(f(t)-g_{1}(t)\right)\left(g_{0}(t)-g_{1}(t)\right)>-K_{f}\left\|f-g_{1}\right\|_{\infty}\left\|g_{0}-g_{1}\right\|_{\infty} .
$$

It can be easily proved that $E\left(f-g_{1}\right)$ is compact.

Then there exists a neighborhood $U$ of $E\left(f-g_{1}\right)$ such that for all $t \in U$,

$$
\left(f(t)-g_{1}(t)\right)\left(g_{0}(t)-g_{1}(t)\right)>-K_{f}\left\|f-g_{1}\right\|_{\infty}\left\|g_{0}-g_{1}\right\|_{\infty}
$$

and

$$
\left|f(t)-g_{1}(t)\right| \geq \frac{1}{2}|| f-g_{1} \|_{\infty} .
$$

Moreover, the neighborhood $U$ can be chosen sufficiently small such that for all $t \in U$ with

$$
\left(f(t)-g_{1}(t)\right)\left(g_{0}(t)-g_{1}(t)\right)<0
$$




$$
\left|g_{0}(t)-g_{1}(t)\right|<K_{f}|| g_{0}-g_{1} \|_{\infty} .
$$

Since $[a, b] \backslash U$ is compact, there exists a real number $c>0$ such that for all $t \in[a, b] \backslash U$,

$$
\left|\left(f(t)-g_{1}(t)\right)\right| \leq|| f-g_{1} \|_{\infty}-c .
$$

By multiplying $\left(g_{0}-g_{1}\right)$ with an appropriate positive factor, assume with out loss of generality that

$$
\left\|g_{0}-g_{1}\right\|_{\infty} \leq \min \left\{c, \frac{1}{2}\left\|f-g_{1}\right\|_{\infty}\right\} .
$$

Case (i) Let $t \in[a, b] \backslash U$. Then

$$
\begin{array}{rlr}
\left|f(t)-g_{0}(t)\right| & \leq\left|f(t)-g_{1}(t)\right|+\left|g_{0}(t)-g_{1}(t)\right| \\
& \leq\left\|f-g_{1}\right\|_{\infty}-c+\left\|g_{0}-g_{1}\right\|_{\infty} \quad \text { by }(3.7) \\
& <\left\|f-g_{1}\right\|_{\infty} \quad \text { by }(3.8)
\end{array}
$$

Case (ii) Let $t \in U$. If $\left(f(t)-g_{1}(t)\right)\left(g_{0}(t)-g_{1}(t)\right)<0$, then it follows that

$$
\begin{aligned}
\left|f(t)-g_{0}(t)\right| & =\left|\left(f(t)-g_{1}(t)\right)-\left(g_{0}(t)-g_{1}(t)\right)\right| \\
& =\left|f(t)-g_{1}(t)\right|+\left|g_{0}(t)-g_{1}(t)\right| \\
& <\left|f(t)-g_{1}(t)\right|+K_{f}|| g_{0}-g_{1} \|_{\infty} \text { by }(3.6) \\
& =\left\|f-g_{1}\right\|_{\infty}+K_{f}\left\|g_{0}-g_{1}\right\|_{\infty} .
\end{aligned}
$$

If $\left(f(t)-g_{1}(t)\right)\left(g_{0}(t)-g_{1}(t)\right) \geq 0$, then it follows that

$$
\begin{aligned}
\left|f(t)-g_{0}(t)\right| & =\left|f(t)-g_{1}(t)-\left(g_{0}(t)-g_{1}(t)\right)\right| \\
& =|| f(t)-g_{1}(t)|-| g_{0}(t)-g_{1}(t)|| \\
& =\left|f(t)-g_{1}(t)\right|-\left|g_{0}(t)-g_{1}(t)\right| \quad \text { by }(3.5) \text { and }(3.8) \\
& <\left|f(t)-g_{1}(t)\right| \\
& =|| f-g_{1} \|_{\infty} .
\end{aligned}
$$

Thus for all $t \in[a, b]$,

$$
\left|f(t)-g_{0}(t)\right|<\left\|f-g_{1}\right\|_{\infty}+K_{f}\left\|g_{0}-g_{1}\right\|_{\infty} .
$$

This implies that $\left\|f-g_{0}\right\|_{\infty}<\left\|f-g_{1}\right\|_{\infty}+K_{f}\left\|g_{0}-g_{1}\right\|_{\infty}$. Which shows that $g_{0}$ is not a strongly unique farthest point to $f$ from $G$, a contradiction.

(iii) $\Rightarrow$ (ii) is obvious. 
(ii) $\Rightarrow$ (iii). Assume that $G \backslash\left\{g_{0}\right\}$ is compact and (ii) holds.

Define a map $F: G \backslash\left\{g_{0}\right\} \rightarrow \mathbb{R}$ by

$$
F(g)=\min _{t \in E(f-g)} \frac{(f(t)-g(t))\left(g_{0}(t)-g(t)\right)}{\|f-g\|_{\infty}\left\|g_{0}-g\right\|_{\infty}} .
$$

It is easy to check that $F$ is continuous. By (ii), $F(g)<0$ for all $g \in G \backslash\left\{g_{0}\right\}$. Since $F$ is continuous on the compact set $G \backslash\left\{g_{0}\right\}$, there exists a constant $K_{f}>0$ such that for all $g \in G \backslash\left\{g_{0}\right\}, F(g)<-K_{f}$.

Thus $\min _{t \in E(f-g)}(f(t)-g(t))\left(g_{0}(t)-g(t)\right) \leq-K_{f}\|f-g\|_{\infty}\left\|g_{0}-g\right\|_{\infty}$.

\section{References}

[1] G.C. Ahuja, T.D. Narang, T. Swaran, On farthest points, Journal of Indian Mathematical Society, 39 (1975), 293-297.

[2] E. Asplund, Farthest points in reflexive locally uniformly rotund Banach spaces, Israel Journal of Mathematics, 4 (1966), 213-216.

[3] M. Edelstein, Farthest points of sets in uniformly convex Banach spaces, Israel Journal of Mathematics, 4 (1966), 171-176.

[4] C. Franchetti, I. Singer, Deviation and farthest points in normed linear spaces, Romanian Journal of Pure and Applied Mathematics, 24 (1979), 373-381.

[5] A.N. Kolmogorov, Eine Bemerkung zu den Polynomen Von P.L. Tschebychelff, die Von einer gegebenen Funktion am Wenigsten abweichen, USP. Math. Nauk, 3 (1948), 216-221.

[6] T.D. Narang, A study of farthest point, New Archive for Mathematics, 25 (1977), 54-79.

[7] B.B. Panda, O.P. Kapoor, On farthest points of sets, Journal of Mathematical Analysis and Applications, 62 (1978), 345-353.

[8] R. Saravanan, Some Contributions to Approximation Theory, Ph.D. Thesis, University of Madras (1997).

[9] I. Singer, Best Approximation in Normed Linear Spaces by Elements of Linear Subspaces, Springer-Verlag, New York (1970). 
